\title{
Mucus Glycoproteins Secreted by Respiratory Epithelial Tissue from Cystic Fibrosis Patients
}

\author{
RALPH C. FRATES, JR., TOKIO T. KAIZU, AND JEROLD A. LAST ${ }^{(25)}$ \\ Departments of Pediatrics and Internal Medicine University of California, Davis, California, USA
}

\begin{abstract}
Summary
Tracheae, bronchi, nasal epithelial, and nasal polyp tissue slices were incubated in tissue culture with $\left[{ }^{3} \mathrm{H}\right]$-glucosamine, and the rate of secretion of labeled mucus glycoproteins was measured. Secretion rates were at least 3- to 6-fold higher for all of the samples from nine patients with cystic fibrosis (CF) who were studied, as compared with values for tissue slices from eight young subjects not affected with this disease. The secreted glycoproteins were further purified into one neutral and three acidic fractions by ion-exchange chromatography on DEAE-cellulose. The glycoproteins secreted by respiratory epithelial tissue from cystic fibrosis subjects contained relatively more of two acidic glycoprotein fractions. Double-label experiments with both $\left[{ }^{3} \mathrm{H}\right]$-glucosamine and $\left[{ }^{35} \mathrm{~S}\right]$-sulfate as mucus glycoprotein precursors further substantiated the shift to more acidic components in the purified mucus glycoproteins and, in addition, suggested a higher level of sulfation of these same two acidic glycoprotein fractions. All four of the labeled glycoprotein fractions secreted by cultured human bronchi cochromatographed with authentic mucus glycoproteins purified from sputum of cystic fibrosis subjects by the same techniques. The differences between mucus glycoproteins from cultured CF airway tissue and mucus glycoproteins from other patients' tissue included relatively increased rates of production, level of sulfation, and greater acidity. Further applications of these in vitro techniques should allow the determination of the enzymatic and biochemical causes of these observed differences in the absence of such potentially confounding variables as concurrent airway infection or of oropharyngeal secretions.
\end{abstract}

\section{Speculation}

The observed differences between the mucus glycoproteins from airway tissue explants of cystic fibrosis patients and the glycoproteins from other patients are consistent with the hypothesis that the lung disease (and possibly other complications) of CF is the result of a genetically determined abnormality of glycoprotein metabolism.

Cystic fibrosis (CF), the most common lethal hereditary disease suffered by Caucasians (6), is usually recognized to be the result of a generalized dysfunction of the exocrine glands $(6,22)$. With respect to pathogenesis, the pancreas and the epithelial cells of the respiratory airways are among the most clinically visible sites of attack. Studies of the differences between tracheobronchial mucus glycoproteins from normal subjects and from patients with cystic fibrosis have enjoyed therefore the attention of several investigators in this field.

Most of our current knowledge of the structure of tracheobronchial mucus glycoproteins from patients with cystic fibrosis has come from studies from the laboratories of Roussel and his colleagues (19) and of Boat et al. (3, 4). Investigations by the former group $(12,20)$ have found more highly sulfated glycoprotein in the sputum of cystic fibrosis patients than in sputum of adults with chronic bronchitis, a result completely in accord with earlier histochemical observations (6). The studies of Boat and his colleagues have also emphasized the shift to more acidic, more highly sulfated glycoproteins in tracheobronchial aspirates or sputum from cystic fibrosis patients $(3,4,5,8)$. Evidence has also been presented that the glycoproteins secreted by cultured nasal polyps from patients with cystic fibrosis are more highly sulfated than are the glycoproteins from patients with allergic rhinitis (2). In addition, Talamo (22) has pointed out the importance of sulfation of mucus glycoproteins as an area in which further research in cystic fibrosis should be directed.

Different aspects of the relationship between altered glycoprotein metabolism and cystic fibrosis have been studied by several other investigators $(1,2,6,10,18,21,22)$. Although not fully elucidated, the role of abnormal glycoproteins should be considered important, if not central, to our understanding of this disease.

A number of methodologies for studying respiratory mucus glycoproteins have evolved. Analysis of sputum or tracheal aspirates has the disadvantage of contamination by oropharyngeal secretions. Analysis of tissue culture secretions avoids contamination, but the small yields of desired specimens have made purification and characterization of mucus glycoproteins difficult. The methodology used in the present study is identical to that currently used for animal studies in our laboratory $(13,14)$. We have studied radionuclide-labeled mucus glycoproteins from rat and monkey tracheal explants extensively $(10,11,16)$, and have found that papain digestion of tissue culture medium, although avoiding contamination by oropharyngeal secretions or products of airway inflammation, yields a sufficient amount of purified mucus glycopeptides to permit their relatively sharp resolution on chromatography. The primary purpose of the present study was to examine possible differences between the respiratory mucus glycoproteins of CF and non-CF subjects. A second objective was to compare our results to those of other workers who have used a different methodology.

\section{MATERIALS AND METHODS}

Human nasal polyps were obtained from excess tissue removed for therapeutic purposes. Nasal epithelial tissue was obtained as a by-product of cosmetic surgery. Parts of tracheae either were removed from cadavers or were removed from patients to permit insertion of a tracheostomy tube. Bronchi were obtained either from cadavers or from excess tissue remaining after a lung resection for therapeutic purposes. Tissue from all of these sources was treated in the same manner. As much of the extraneous vascular and other adventitious tissue as possible was trimmed off. The tissue was then immersed immediately into a vial of Dulbecco's modified Eagle's Medium (GIBCO) for transport to the laboratory. In general, tissue was delivered to the laboratory within 1-2 $h$ of its removal from an individual, though longer times of storage in culture medium are apparently not deleterious to these assays. At all times the temperature of the tissue slice was maintained as close to $37^{\circ} \mathrm{C}$ as was feasible. As shown in Figure 1, the amount of labeled glycoprotein secreted under standard assay conditions is proportional to the size of the slice tested, within experimental error. The difficulties in accurately slicing and/or measuring tissue 


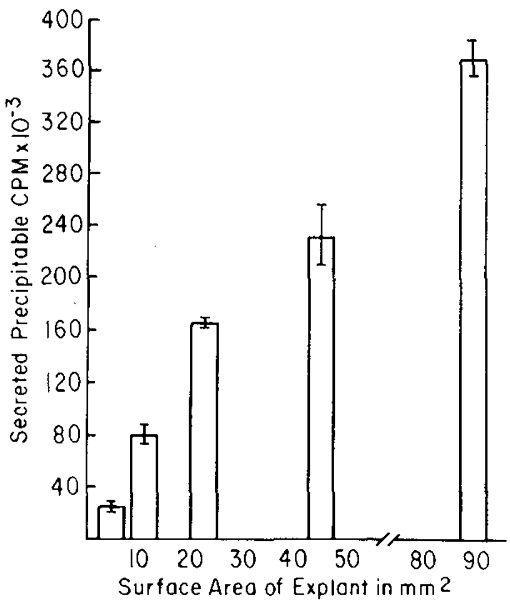

Fig. 1. Glycoprotein secretion rate by different sized tracheal slices from rhesus monkey incubated for $24 \mathrm{~h}$ in culture. Incorporation is linear for slices larger than $20 \mathrm{~mm}^{2}$, as shown.

smaller than $25 \mathrm{~mm}^{2}$, the surface area above which the incorporation rate was linear with tissue size (Figure 1), suggests that, where possible, slices should be at least $25 \mathrm{~mm}^{2}$ for careful experimentation. In general, adequate amounts of bronchi and tracheae were available to allow the use of slices of $70-75 \mathrm{~mm}^{2}$, but only enough nasal epithelial or polyp tissues were obtained to allow slices of $5-20 \mathrm{~mm}^{2}$ to be prepared. Not surprisingly, the tissue of which the smallest amount was available was nasal epithelial.

\section{RESULTS}

Rate of secretion of mucus glycoproteins by tissue slices. Respiratory epithelial tissues have been cultured thus far from nine patients with cystic fibrosis and from eight subjects not affected with this disease. At culture, the investigator did not know the diagnosis; samples were coded. The results of these studies are summarized in Table 1 . Several points are immediately apparent upon inspection of these data. First, within any given tissue type, explants from patients with cystic fibrosis secrete glycoproteins at a more rapid rate than do explants of the same surface area from control subjects. The observed rates are 3- to 4-fold higher with tracheae and 4- to 6-fold higher with bronchi. Too few samples (thus far) and uncertainties with regard to the exact surface area (see above) of nasal epithelial tissue make quantitative comparisons more difficult, but the data from these nasal tissues agree qualitatively with this generalization. Second, the measured net secretion rates are relatively consistent for a given type of tissue from either a single individual (low coefficient of variance in the assay) or from a different matched individual (provided either another cystic fibrosis patient or another non-cystic fibrosis subject is being compared). Third, nasal polyps, which can be obtained with minimal trauma to the individual donor, mirror the quantitative changes apparent in this assay occurring in the trachea and bronchi of an individual affected with cystic fibrosis. Fourth, the various epithelial tissue explants mirror in vitro the fundamental lung lesion of cystic fibrosis patients in vivo: excessive production of mucus glycoproteins by the respiratory airway(s). Finally, these tissue explants produce enough labeled glycoprotein in culture to allow further studies of the composition and structure to be performed, thus allowing putative qualitative changes (as well as quantitative) to be evaluated in the cystic fibrosis mucus glycoproteins.

Separation and purification of secreted labeled $\left[{ }^{3} \mathrm{H}\right]-$ and $\left[{ }^{35} \mathrm{~S}\right]-$ glycopeptides. The mucus glycoproteins labeled with $\left[{ }^{3} \mathrm{H}\right]$-glucosamine and/or with $\left[{ }^{35} \mathrm{~S}\right]$-sulfate from selected samples in Table 1 were further studied by chromatography on DEAE-cellulose after papain solubilization. Figure 2 illustrates the elution profile of $\left[{ }^{3} \mathrm{H}\right]-$ and $\left[{ }^{35} \mathrm{~S}\right]$-labeled glycopeptides from such a column in which the culture medium from a bronchial explant from a non-cystic fibrosis subject was analyzed. The neutral glycopeptide fraction, centered at tube No. 3 , is eluted as a single peak of unbound $\left[{ }^{3} \mathrm{H}\right]$ radioactivity, the exact shape of which is mainly determined by the ratio of the initial volume in which the sample is applied to the ratio of the volume of the fractions collected. This peak is not detectable when $\left[{ }^{35} \mathrm{~S}\right]$-sulfate is used as the radioactive precursor of the mucus glycoproteins. After the neutral fraction is completely eluted, the acidic glycopeptides are eluted with a linear salt gradient from 0 to $0.67 \mathrm{M}$ lithium chloride. Under these conditions

Table 1. Mucus glycoprotein secretion rates of human airway explants ${ }^{1}$

\begin{tabular}{|c|c|c|c|}
\hline $\begin{array}{c}\text { Experiment } \\
\text { no. }\end{array}$ & Tissue source & Patient's status & $\begin{array}{c}\text { Glycoprotein } \\
\text { secretion rate } \\
{[\mathrm{cpm} \text { secreted/ }} \\
\left.22 \mathrm{~h}\left(\times 10^{-3}\right)\right]^{2}\end{array}$ \\
\hline 1 & Tracheae & Normal & $9.6 \pm 1.8(6)$ \\
\hline 2 & Tracheae & Normal & $9.7 \pm 0.4(7)$ \\
\hline 3 & Tracheae & Cystic fibrosis & $32.1 \pm 3.2(6)$ \\
\hline 4 & Tracheae & Cystic fibrosis & $28.1 \pm 6.0(6)$ \\
\hline 5 & Tracheae & Cystic fibrosis & $40.1 \pm 9.2(6)$ \\
\hline 6 & Bronchi & Normal & $18.7 \pm 2.4(5)$ \\
\hline 7 & Bronchi & Normal & $36.1 \pm 2.5(3)$ \\
\hline 8 & Bronchi & Normal & $28.0 \pm 3.0(6)$ \\
\hline 9 & Bronchi & Cystic fibrosis & $180.3 \pm 20.2(5)$ \\
\hline 10 & Bronchi & Cystic fibrosis & $154.8 \pm 15.1(5)$ \\
\hline 11 & Bronchi & Cystic fibrosis & $121.0 \pm 19.7(6)$ \\
\hline 12 & Nasal polyp & Normal & $4.4 \pm 0.6(4)$ \\
\hline 13 & Nasal polyp & Cystic fibrosis & $84.2 \pm 9.1(5)$ \\
\hline 14 & Nasal polyp & Cystic fibrosis & $54.8 \pm 4.7(6)$ \\
\hline 15 & Nasal epithelium & Normal & $97.0 \pm 6.2(5)$ \\
\hline 16 & Nasal epithelium & Normal & $21.0(1)$ \\
\hline 17 & Nasal epithelium & Cystic fibrosis & $156.4 \pm 9.4(5)$ \\
\hline
\end{tabular}

${ }^{1}$ Each experiment number corresponds to tissue from a different individual. $\left[{ }^{3} \mathrm{H}\right]-$ Glucosamine was used as a precursor in all of the cited studies $(2 \mu \mathrm{Ci} / \mathrm{ml}, 8-10 \mathrm{Ci} / \mathrm{mmole})$.

${ }^{2}$ Mean \pm S.E. (number of slices assayed). Tracheae and bronchi were $70-75 \mathrm{~mm}^{2}$ surface area, whereas nasal polyps were cut to about $15 \mathrm{~mm}^{2}$ and nasal epthelial tissue was $\leq 15 \mathrm{~mm}^{2}$.

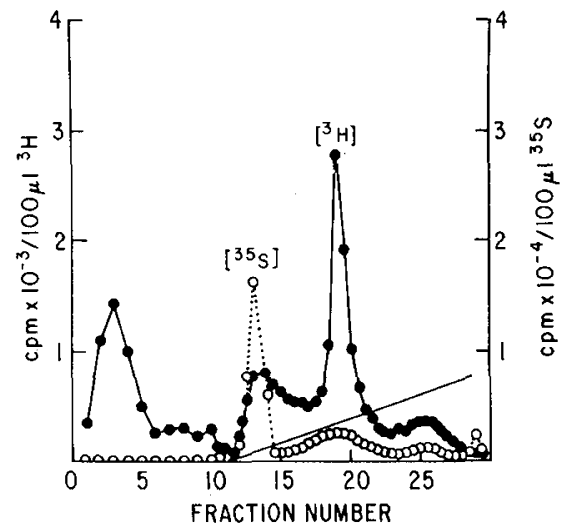

Fig. 2. Chromatography on DEAE-cellulose of labeled mucus glycopeptides secreted by cultured human bronchial tissue from a non-cystic fibrosis subject. Labeled precursors used were $\left[{ }^{3} \mathrm{H}\right]$-glucosamine and $\left[{ }^{35} \mathrm{~S}\right]$ -sulfate $\left(\mathrm{O}_{-}\right)$, elution profile of $\left[{ }^{35} \mathrm{~S}\right]$-glycopeptides from the column and $(-), \mathrm{LiCl}$ salt gradient used for elution. Fractions of $3.4 \mathrm{ml}$ were collected until fraction No. 12; fractions of $1.7 \mathrm{ml}$ were collected subsequently. Fraction numbers are assigned on the basis of $3.4 \mathrm{ml}$ fractions (i.e., there are two data points for each fraction in the acidic region). Starting buffer used was $0.01 \mathrm{M} \mathrm{LiCl}$ in $50 \mathrm{mM}$ acetate, $\mathrm{pH} 4.4$. 
three acidic glycopeptide fractions are obtained (Fig. $2,\left[{ }^{3} \mathrm{H}\right]-$ labeled trace), centered at fractions Nos. 13-14, 19, and 26. The first of these acidic peaks is labeled with $\left[{ }^{35} \mathrm{~S}\right]$ from precursor sulfate. The two more acidic fractions (at No. 19 and at 26) are reproducibly labeled to a relatively small extent with $\left[{ }^{35} \mathrm{~S}\right]$-sulfate with respect to the first acidic fraction (at No. 13-14) when noncystic fibrosis subjects are studied. The second acidic fraction is quantitatively the most important acidic fraction as defined by $\left[{ }^{3} \mathrm{H}\right]$-glucosamine incorporation.

It is not possible to draw quantitative conclusions about the actual amounts of sulfate and of glucosamine being incorporated into these peaks as we do not know the relevant pool sizes of these substances within the cells that actually synthesize the mucus glycoproteins. If we assume that equivalent precursor pools exist in normal and cystic fibrosis bronchial tissue, however, we can examine the relative ratio of $\left[{ }^{35} \mathrm{~S}\right]$ to $\left[{ }^{3} \mathrm{H}\right]$ within each peak $(2,8)$ and draw tentative conclusions with regard to the average level of sulfation of the glycoproteins within each fraction. Figure 3 illustrates an analogous experiment to that of Figure 2, except that the bronchial explant is from a resected lung from a patient with cystic fibrosis. In common with the tissue from a non-cystic fibrosis subject, a single peak of neutral glycopeptide labeled only with $\left[{ }^{3} \mathrm{H}\right]$-glucosamine is observed. The apparent additional peak at fractions No. 4-6 is an artifact of how the sample was applied. The apparent low level of $\left[{ }^{35} \mathrm{~S}\right]$-sulfate labeling of the neutral fraction is probably accounted for by contamination of this fraction with a small amount of acidic glycopeptide, although we cannot at this time rule out very low levels of sulfation of the neutral fraction from cystic fibrosis explants. The low level of $\left[{ }^{35} \mathrm{~S}\right]$ appearing in the neutral fraction from this chromatographic column has not been a reproducible finding.

Even more interesting, however, is the appearance of the acidic fractions from cystic fibrosis tissue. Three peaks of $\left[{ }^{3} \mathrm{H}\right]$-glucosamine-labeled glycopeptides are again observed. These peaks are centered at fractions Nos. 13, 19, and 27-29, apparently comparable to the corresponding fractions from normal bronchial explants. When we examine the elution pattern of $\left[{ }^{35} \mathrm{~S}\right]$-sulfatelabeled glycopeptides, however, we observe dramatic differences between the normal and cystic fibrosis products. The most striking difference is the very high level of incorporation of $\left[{ }^{35} \mathrm{~S}\right]$ into the second and third acidic fractions, relative to incorporation into the first fraction as was observed in the normal bronchial explants. Although the ratio of incorporation of $\left[{ }^{35} \mathrm{~S}\right]$ to $\left[{ }^{3} \mathrm{H}\right]$ into the first acidic peak appears similar as plotted (note change of scales) when bronchial explants from normal and cystic fibrosis subjects are compared, the relative incorporation of $\left[{ }^{35} \mathrm{~S}\right]$ to $\left[{ }^{3} \mathrm{H}\right]$ is increased some 10 - to 15 -fold into the second and third acidic peaks. Assuming equivalence of precursor pools (see above), the second and third acidic glycopeptide fractions must be relatively more highly sulfated in the secretory products from cystic fibrosis bronchi than from normal bronchi.

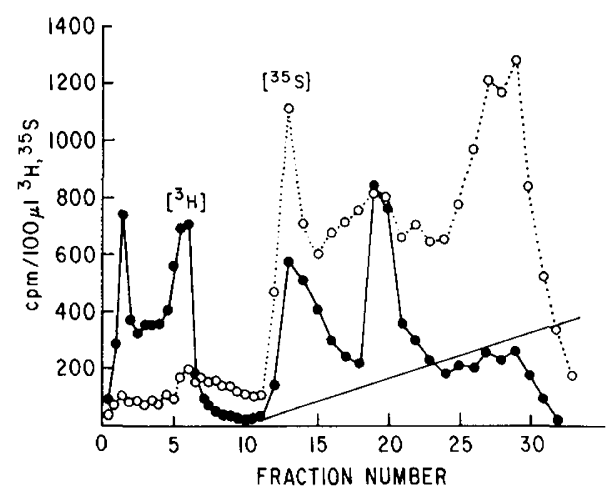

Fig. 3. Chromatography on DEAE-cellulose of labeled mucus glycopeptides secreted by cultured bronchial tissue from a cystic fibrosis subject. Details as in Figure 2. Note that the scale used for $\left[{ }^{3} \mathrm{H}\right]$ is ten times greater than for $\left[{ }^{35} \mathrm{~S}\right]$.

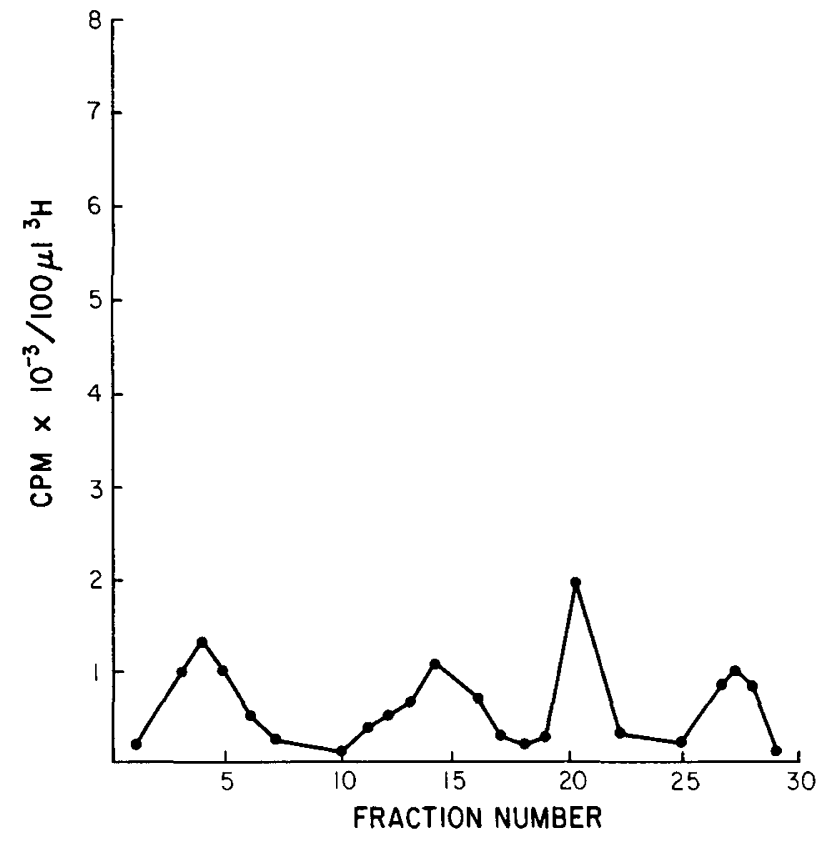

Fig. 4. Chromatography on DEAE-cellulose of labeled mucus glycopeptides secreted by cultured bronchial tissue from a patient with agammaglobulinemia (see Experiment No. 6, Table 1).

The relative distribution of the $\left[{ }^{3} \mathrm{H}\right]$-glucosamine-labeled glycopeptides seen in Figure 4 are from bronchial explants of an agammaglobulinemic patient with purulent bronchitis (Experiment 6, Table 1). Upon comparison with other "normal" bronchial explants (Fig. 2), note that the elution profile is also essentially normal as compared with the acidic fractions from cystic fibrosis tissue.

Comparison of secreted glycopeptides and glycopeptides from sputum. Sputum collected by expectoration from a patient with cystic fibrosis was washed exhaustively with $0.15 \mathrm{M} \mathrm{NaCl}$ (3-5 washings of $\mathrm{ml}$ each, followed by collection of the gel phase by centrifugation at $8000 \mathrm{rpm}$ for $10 \mathrm{~min}$ in a Sorvall SS-32 rotor). The gel was resuspended in Tris- $\mathrm{HCl}$ buffer by vortex mixing; and. the resultant suspension was treated with papain, dialyzed, and lyophilized, as described in the "Materials and Methods" section for labeled glycoproteins from tracheal explant culture medium. The resultant solubilized glycopeptide preparation was chromatographed on a DEAE-cellulose column similar to that used for the labeled glycopeptides from papain-digested airway tissue specimens. The resultant elution pattern (7) and that of the glycopeptides secreted by the bronchial explants from a cystic fibrosis patient (Fig. 3) were similar (data not shown). These results suggest that the glycoproteins secreted by airway explants from patients with cystic fibrosis accurately mimic the composition of the tracheobronchial glycoproteins being synthesized in vivo.

\section{DISCUSSION}

The experiments reported in this paper demonstrate that airway explants from cystic fibrosis patients, as compared with those from non-cystic fibrosis subjects: (1) make mucus glycoproteins at a faster rate in culture (Table 1); (2) make a mixture of glycopeptides in which two of the four peaks separated on DEAE-cellulose are apparently more heavily sulfated (Figs. 2 and 3); and (3) make a fraction showing augmented sulfation (fractions Nos. 19-26 in Fig. 3). It is important to emphasize that there are conceptual and technical differences in our approach to these studies as compared with earlier work in this area. These results not only confirm and extend previous reports using different methodology, but also allow several novel conclusions to be drawn. Our discussion of these results is keyed to the findings enumerated above.

The first major methodologic difference between our studies 
and those of Boat and co-workers $(2,5)$ relates to the duration of incubation of tissue in culture medium. We have focused our attention on short-term $(22 \mathrm{~h})$ incubation of tissue as opposed to the 7-10 days, which is customarily used by others. Our rationale for this was that Boat et al. have shown a decrease in the rate of secretion of glycoproteins after one day in culture for nasal polyps (2) and for tracheal tissue (5), which we wanted to avoid so as to enable rates of secretion to be directly compared between experiments, although we should point out that the time course of glycoprotein secretion apparently remains linear for at least 72-96 $\mathrm{h}$ in our system thus allowing rates to be determined as the slope of such lines over this entire interval $(10,15)$. In addition, shortterm incubations in culture seem more likely to accurately mirror the metabolic capacities of the tissue in vivo. This assumption is especially likely because we have shown elsewhere that there is a shift towards the production of relatively more acidic mixtures of mucus glycoproteins with longer durations of incubation of tracheal tissue in vitro from various experimental animals (11). It is worthwhile to point out the dissimilarities between our work and that of Neutra et al. (17). Those investigators used autoradiography to determine the distribution and transit time of labeled glycoproteins within human rectal epithelium. They found no differences in their in vitro assay of rectal biopsy explants between cystic fibrosis patients and biopsies from volunteers who did not have cystic fibrosis. But autoradiography does not allow quantitation of glycoprotein synthesis or secretion (as precursor pool sizes were not determined), nor does their method allow separation of glycoproteins (glycopeptides) by chromatographic analysis of secretory products available to us. Furthermore, there probably are differences between gut and airway epithelial mucus glycoprotein metabolism even in cystic fibrosis patients. These metabolic differences would further confound comparison of Neutra et al.'s results (17) to the present study. It should be noted that neither our experiments nor the investigations of Boat et al. (5) distinguish between possible increased rate of glycoprotein secretion by individual cells due to intrinsic cellular differences versus increased glycoprotein secretion secondary to hypertrophy and hyperplasia of mucus-secreting cells.

Because of these differences in experimental design from those of previous workers, we were encouraged to compare the net secretion rate in vitro under our experimental conditions for the various tissues studied (Table 1). Two conclusions may be drawn from these data. First, tissue samples from cystic fibrosis subjects secrete mucus glycoproteins at a faster rate in vitro than do samples of equal surface area from non-cystic fibrosis subjects. This conclusion might have been predicted from the clinical symptoms of the disease (i.e., mucus hypersecretion), but to our knowledge this is the first systematic comparison of a series of explants that has documented such an increased rate of glycoprotein secretion by tissue from cystic fibrosis subjects in vitro. Because the tissue explants are cultured under aseptic conditions in the presence of antibiotics, we can rule out a direct role of concurrent bacterial infection in the observed augmented rate of glycoprotein secretion.

The second conclusion that may be drawn is that similar changes are seen with cultured bronchi, with cultured tracheae, with cultured nasal epithelial tissue, and with cultured nasal polyps. In all tissue types studied, the rate of secretion of glycoproteins by the cultured tissue from cystic fibrosis subjects was apparently much more rapid than with tissue from unaffected subjects, as shown in Table 1 . The altered rate of secretion observed fulfills one of the minimal criteria for a genetic defect: it is expressed in several different tissues of the respiratory epithelium. This was not a result that would necessarily have been predicted on the basis of the existing literature. It is also uncertain whether these changes occur in nonrespiratory epithelial tissue (17); further studies in this area, especially with gastrointestinal mucosal explants in culture, are necessary.

The apparently more heavily sulfated acidic glycoprotein mixture from cystic fibrosis explants (compare Figs. 2 and 3) was an expected finding based on the previously reported histochemical (6) and biochemical $(2,3,4,5,12,19)$ results of others; however, another technical difference between the experiments reported herein and those of others should be noted. The use of papain treatment to solubilize mucus glycoproteins rather than reduction and alkylation of sulfhydryl residues with iodoacetate $(2,3,4,5$, 19) seems to result in a sharper separation of glycopeptides into discrete fractions upon ion-exchange columns. In addition, the yields upon chromatography after papain treatment are better (4, 9 ); they are essentially quantitative in our hands. We also find better resolution of the acidic glycopeptide fractions by gradient elution from DEAE-cellulose than by the batch elution techniques used by others $(2,3,4,5,12,19,20)$. It should also be emphasized that we have shown elsewhere (11) that no detectable glycosaminoglycans appear in the papain-treated culture medium from tracheal or bronchial slices incubated under these conditions.

The role of intercurrent lung infections in the genesis of changes in the mucus glycoproteins of cystic fibrosis subjects has been a source of concern for several investigators in this field $(5 ; 19)$. Use of cultured airway explants, as has been pointed out previously $(2,5)$, allows the investigator to bypass some of the difficulties of dealing with fluids from purulent, infected samples of airway mucus glycoproteins such as are obtained from sputum and from tracheobronchial washings. The explants can be cultured aseptically in the presence of sterilizing amounts of antibiotics to avoid this source of complication. Nor is bronchorrhea from infection sufficient explanation for our findings. Sample No. 6 of Table 1 was taken from a 15 -year-old boy who underwent a pneumonectomy for treatment of complications of agammaglobulinemia. His lungs were continuously infected with Hemophilus influenza and he produced several ounces of viscid, purulent sputum every day. Nevertheless, his airway explants did not demonstrate the enhanced rate of secretion of glycoproteins characteristic of the cystic fibrosis tissue, nor were the resultant mucus glycopeptides produced more heavily sulfated upon DEAE-cellulose chromatography (Fig. 4). We can tentatively conclude that the presence of recurrent lung infection (of the small airways) is not by itself a sufficient explanation for the enhanced rate of mucus glycoprotein secretion by airway explants from cystic fibrosis subjects. Because we saw no indication of augmented acidic fractions in the sample from the boy with agammaglobulinemia (Fig. 4), we also conclude that these changes observed with cystic fibrosis explants (Fig. 3) are not caused by the intercurrent infection(s) in vivo.

Our current efforts are directed towards further characterization of, and structural studies upon, the labeled glycoprotein fractions prepared by DEAE-cellulose column chromatography. Although dealing with such minute amounts of labeled glycoprotein (pmole quantities) is extremely difficult, we feel that the ultimate definition of the structure of mucus glycoproteins secreted by cystic fibrosis and by normal airway tissue must come from studies in tissue culture. Only with cultured tissue may one circumvent the problems caused by potential interference and/or complications of interpretation introduced by chronic infection, inflammation, and the presence of proteins and fluids arising from sources other than respiratory epithelial tissue such as occur when the source of glycoproteins studied is either sputum or tracheobronchial washings.

\section{REFERENCES AND NOTES}

1. Alhadeff, J. A.: Glycoproteins and cystic fibrosis: A review. Clin. Genet., 14: 189 (1978)

2. Boat, T. F., Kleinerman, J. I., Carlson, D. M., Maloney, W. H., and Matthews, L. W.: Human respiratory tract secretions. I. Mucous glycoproteins secreted by cultured nasal polyp epithelium from subjects with allergic rhinitis and with cystic fibrosis. Amer. Rev. Resp. Dis., 110: 428 (1974).

3. Boat, T. F., Cheng, P. W., Iyer, R. N., Carlson, D. M., and Polony, I.: Human respiratory tract secretions. Mucus glycoproteins of nonpurulent tracheobronchial secretions, and sputum of patients with bronchitis and cystic fibrosis. Arch. Biochem. Biophys., 177: 95 (1976).

4. Boat, . F. and Cheng, P. W.: Mucous glycoproteins. In: J. A. Mangos and R. C. Talamo, Eds.: Cystic Fibrosis: Projections into the Future. pp. 165-177 (Stratton.Intercontinental Medical Book Corp., New York, 1976).

5. Boat, T. F., Cheng, P. W., and Wood, R. E.: Tracheobronchial mucus secretion in vivo and in vitro by epithelial tissues from cystic fibrosis and control subjects. Mod. Probl. Pediatr., 19: 14l (1977). 
6. DiSant'Agnese, P. A. and Davis, P. B.: Research in cystic fibrosis. New Engl. J. Med., 295: 481-485, 534-541, 597-602 (1976).

7. Dubois, M., Gilles, K. A., Hamilton, J. K., Rebers, P. A., and Smith, F Colorimetric method for determination of sugars and related substances. Anal. Chem., 28: 350 (1956).

8. Gallagher, J. T. and Kent, P. W.: Structure and metabolism of glycoproteins and glycosaminoglycans secreted by organ cultures of rabbit trachea. Biochem. J., 148: 187 (1975).

9. Hallen, A.: Chromatography of acidic glycosaminoglycans on DEAE-cellulose. J. Chromatogr., 71: 83 (1972).

10. Jennings, M., Cross, C. E., and Last, J. A.: Glycoprotein synthesis by trachea explants from various mammalian species. Comp. Biochem. Physiol., 57A: 317 (1977).

11. Kaizu, T., Lyons, S. A., Cross, C. E., Jennings, M. D., and Last, J. A.: Composition of glycoproteins secreted by tracheal explants from various animal species. Comp. Biochem. Physiol., 62B: 195 (1979).

12. Lamblin, G., Lafitte, J. J., Lhermitte, M., Degand, P., and Roussel, P.: Mucins from cystic fibrosis sputum. Mod. Probl. Pediatr., 19: 153 (1977).

13. Last, J. A. and Cross, C. E.: A new model for health effects of air pollutants: Evidence for synergistic effects of mixtures of ozone and sulfuric acid aerosols on rat lungs. J. Lab. Clin. Med., 91: 328 (1978).

14. Last, J. A., Jennings, M. E., and Moore, P. F.: Chromate inhibition of metabolism by rat tracheal explants. Lab. Invest., 37: 276 (1977)

15. Last, J. A., Jennings, M., Schwartz, L. W., and Cross, C. E.: Glycoprotein secretion by tracheal explants cultured from rats exposed to ozone. Amer. Rev. Resp. Dis., 116: 695 (1977).

16. Last, J. A. and Kaizu, T.: Mucus glycoprotein secretion by tracheal explants: Effects of pollutants. Environ. Health Perspect., 35: 131 (1980).

17. Neutra, M. R., Grand, R. J., and Trier, J. S.: Glycoprotein synthesis, transport, and secretion by epithelial cells of human rectal mucosa. Lab. Invest., $36: 535$ (1977).

18. Rao, G. J. S., Spells, G., and Nadler, H. L.: Enhanced UDP-galactose: glycoprotein galactosyl transferase activity in cultivated skin fibroblasts from patients with cystic fibrosis and its possible relationship to the pathogenesis of the disease. Pediatr. Res., 11: 981 (1977)

19. Roussel, P., Lamblin, G., Degand, P., Walker-Nasir, E., and Jeanloz, R. W. Heterogeneity of the carbohydrate chains of sulfated bronchial glycoproteins isolated from a patient suffering from cystic fibrosis. J. Biol. Chem., 250: 2114 (1975).

20. Roussel, P., Degand, P., Lamblin, G., Laine, A., and Lafitte, J. J.: Biochemical definition of human tracheobronchial mucus. Lung, 154: 241 (1978).

21. Scanlin, T. F.: Abnormal distribution of $\alpha$-L-fucosidase in cystic fibrosis: Increased activity in skin fibroblasts. Biochem. Biophys. Res. Commun., 79: 869 (1977).

22. Talamo, R. C.: Summary of "fundamental sessions". In: J. A. Mangos and R. C. Talamo, Eds.: Cystic Fibrosis: Projections into the Future. pp. 351-356 (Stratton Intercontinental Medical Book Corp., New York, 1976).

23. Informed consent was solicited at all times when tissue was obtained as a byproduct of a surgical procedure. All protocols for tissue acquisition were approved by the University Committee on the Ethics of Human Experimentation.

24. This research was supported in part by NIH grants RR-00169 and HL-17957, by EPA Grant R-805355, and by a grant from the California Lung Association.

25. Requests for reprints should be addressed to: Jerold A. Last, Ph.D., California Primate Research Center, University of California, Davis, California 95616 (USA).

26. Received for publication August 13, 1981

27. Accepted for publication May 9, 1982. 